\title{
Non-epidermolytic palmoplantar keratoderma
}

INSERM

\section{Source}

INSERM. (1999). Orphanet: an online rare disease and orphan drug data base. Nonepidermolytic palmoplantar keratoderma. ORPHA:2337

Non-epidermolytic palmoplantar keratoderma is characterised by a diffuse nonepidermolytic palmoplantar keratoderma with frequent fung al infections. Prevalence in the general population is estimated at 1 in 40,000 but is much higher in northern Sweden (0.3-0.55\%). Transmission is autosomal dominant and the causative gene has been localised to chromosome 12q11-q13. 